\title{
A Multi-MW Proton/Electron Facility at KEK
}

\author{
R. Belusevic \\ IPNS, High Energy Accelerator Research Organization (KEK), Ibaraki, Japan \\ Email: belusev@post.kek.jp
}

How to cite this paper: Belusevic, R. (2017) A Multi-MW Proton/Electron Facility at KEK. Journal of Applied Mathematics and Physics, 5, 1222-1242.

https://doi.org/10.4236/jamp.2017.56105

Received: February 10, 2017

Accepted: June 6, 2017

Published: June 9, 2017

Copyright $\odot 2017$ by author and Scientific Research Publishing Inc. This work is licensed under the Creative Commons Attribution International License (CC BY 4.0).

http://creativecommons.org/licenses/by/4.0/

\begin{abstract}
The main "bottleneck" limiting the beam power in circular machines is caused by space charge effects that produce beam instabilities. To increase maximally the beam power of a "proton driver", it is proposed to build a facility consisting solely of a $2.5 \mathrm{GeV}$ injector linac (PI) and a $20 \mathrm{GeV}$ pulsed superconducting linac (SCL). Such a facility could be constructed using the existing KEK accelerator infrastructure. The PI, based on the European Spallation Source (ESS) linac, would serve both as an injector to the SCL and a source of proton beams that could be used to copiously produce, e.g., muons and "cold" neutrons. Protons accelerated by the SCL would be transferred through the KEK Tristan ring in order to create neutrino, kaon and muon beams for fixed-target experiments. At a later stage, a $70 \mathrm{GeV}$ proton synchrotron could be installed inside the Tristan ring. The SCL, comprising 1.3 GHz ILC-type rf cavities, could also accelerate polarized or unpolarized electron beams. After acceleration, electrons could be used to produce polarized positrons, or may traverse an XFEL undulator.
\end{abstract}

\section{Keywords}

Proton Driver, Superconducting Linac, Neutrino Oscillations, Rare Kaon Decays, XFEL

\section{Introduction}

The Standard Model (SM) of particle physics is supported by two theoretical "pillars": the gauge principle and the Higgs mechanism for particle mass generation. In this theoretical model, the mass of a particle depends on its interaction with the Higgs field, a medium that permeates the universe. The SM predicts the existence of a neutral spin-0 particle (the Higgs boson) associated with the Higgs field, but it does not predict its mass. Whereas the gauge principle has been firmly established through precision electroweak measurements, the Higgs mechanism is yet to be fully tested. 
The Higgs-boson mass, $m_{H}$, affects the values of electroweak observables through radiative corrections. Many of the electroweak measurements obtained so far may be combined to provide a global test of consistency with the SM. The best constraint on $m_{H}$ is obtained by making a global fit to the electroweak data. Such a fit is consistent with the value of the Higgs mass measured at the Large Hadron Collider (LHC) [1] [2].

To discover a new particle (such as the Higgs boson), or to search for physics beyond the SM, usually requires the use of high-energy hadron or electron-positron colliders. However, many important discoveries in particle physics have been made using proton beams with relatively low energies but high intensities (flavor mixing in quarks and in neutrinos are noteworthy examples). Experiments with high-intensity neutrino beams, e.g., are designed primarily to explore the mass spectrum of the neutrinos and their properties under the CP symmetry.

Some of the most important discoveries emerged from high-precision studies of K mesons ("kaons"), in particular neutral kaons. A deeper insight into CP violation is expected to be gained from measurements of ultra-rare kaon decays such as $K_{\mathrm{L}}^{0} \rightarrow \pi^{0} \nu \bar{\nu}$ and $K_{\mathrm{L}}^{+} \rightarrow \pi^{+} \nu \bar{\nu}$. These decays provide important information on higher-order effects in electroweak interactions, and therefore can serve as a probe of new phenomena not predicted by the Standard Model.

The physics programs envisaged at the proposed facility are, to a large extent, complementary to each other. For instance, neutrino oscillation experiments and searches for permanent electric dipole moments both look for new sources of $\mathrm{CP}$ violation, a phenomenon which reflects the fundamental difference between matter and antimatter.

A unique feature of the proposed facility is the use of superconducting ILCtype cavities to accelerate both protons and electrons, which considerably increase its physics potential. Polarized electrons and positrons can be used to study the structure of composite particles and the dynamics of strong interactions, as well as to search for new physics beyond the Standard Model.

\section{The Proposed Proton/Electron Facility at KEK}

The main "bottleneck" limiting the beam power in circular machines is caused by space charge effects that produce beam instabilities. Such a "bottleneck" exists at the J-PARC proton synchrotron complex, and is also intrinsic to the "proton drivers" envisaged at CERN and Fermilab. To increase maximally the beam power of a "proton driver", it is proposed to build a facility consisting solely of a low-energy injector linac and a high-energy pulsed superconducting linac. Pulsed operation is preferred over the CW mode (continuous wave, 100\% duty) mainly because the former allows the use of rf cavities with high accelerating gradients. This would considerably reduce the overall length of the machine, which is limited by the size of the KEK site.

The layout of the proposed proton/electron facility at KEK is shown in Figure 1. A $2.5 \mathrm{GeV}$ proton linac (PI) serves both as an injector to a superconducting 


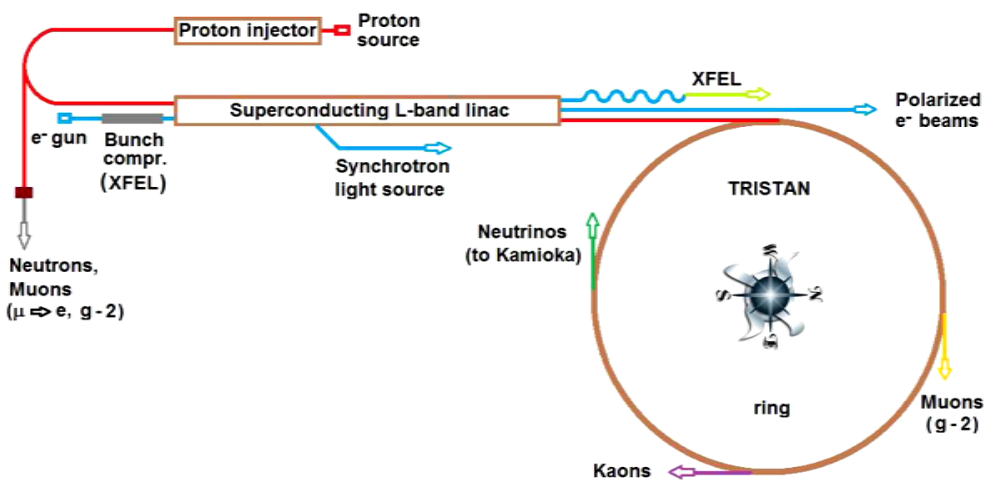

Figure 1. The layout of the proposed multi-purpose proton/electron facility at KEK. The Tristan ring would initially be equipped only with magnets capable of steering the proton bunches, accelerated by the superconducting linac, to various fixed targets.

linac (SCL) and a source of proton beams that can be used to copiously produce neutrons and muons. Protons accelerated by the SCL to $20 \mathrm{GeV}$ are transferred through the KEK Tristan ring in order to create beams for various fixed-target experiments. At a later stage, a $70 \mathrm{GeV}$ proton synchrotron could be installed inside the Tristan ring. The SCL, comprising $1.3 \mathrm{GHz}$ superconducting ILC-type rf cavities, can also accelerate polarized or unpolarized electron bunches. After acceleration, electrons may be used to produce polarized positrons. An SCL-based XFEL and a synchrotron light source for applications in materials science and medicine are also envisaged.

The proposed facility could be constructed using the existing KEK accelerator infrastructure. As shown in Figure 2, the present KEK linac tunnel and klystron gallery could be extended to increase the length, and hence the maximum energy, of each linac in Figure 1. The cryomodules, RF sources and cryogenic plant units of the proposed linac complex would be installed inside these extended structures (see Figure 3). The Tristan ring (TR) would initially be equipped only with magnets capable of steering the SCL proton bunches to various fixed targets. The four $200 \mathrm{~m}$-long straight sections of the TR, each with an experimental hall in the middle (see Figure 2), would house beam lines and detectors.

The beam power of a pulsed linear accelerator is given by the expression

$$
P_{\mathrm{b}}[\mathrm{MW}]=\mathrm{E}_{\mathrm{b}}[\mathrm{MV}] \times I[\mathrm{~A}] \times \tau_{\mathrm{p}}[\mathrm{s}] \times R[\mathrm{~Hz}]
$$

where $P_{\mathrm{b}}$ is the beam power, $\mathrm{E}_{\mathrm{b}}$ is the beam energy, $I$ is the average current per pulse, $\tau_{\mathrm{p}}$ is the beam pulse length, and $R$ is the repetition rate. The duty cycle of a pulsed linac is $D \equiv \tau_{\mathrm{p}} R$. Using the values from Table 1 , and assuming $\mathrm{E}_{\mathrm{b}}=20 \mathrm{GeV}$, one obtains $D=0.024$ and

$$
P_{\mathrm{b}}=20000 \mathrm{MV} \times 31 \mathrm{~mA} \times 1.2 \mathrm{~ms} \times 20 \mathrm{~s}^{-1} \approx 15 \mathrm{MW}
$$

The beam parameters in Table 1 are mutually constrained by the following relations: The number of protons per second $N=P_{\mathrm{b}} / \mathrm{E}_{\mathrm{b}}$ and the number of protons per pulse $N_{\mathrm{p}}=N / R$; the average current per pulse is $I \equiv\left(N_{\mathrm{p}} \times 1.6 \times 10^{-19} \mathrm{C}\right) / \tau_{\mathrm{p}}$. The klystron pulse length is the sum of the rf cavity fill time (current dependent) and the beam pulse length: $\tau=\tau_{\mathrm{f}}+\tau_{\mathrm{p}}$. For ILC- 


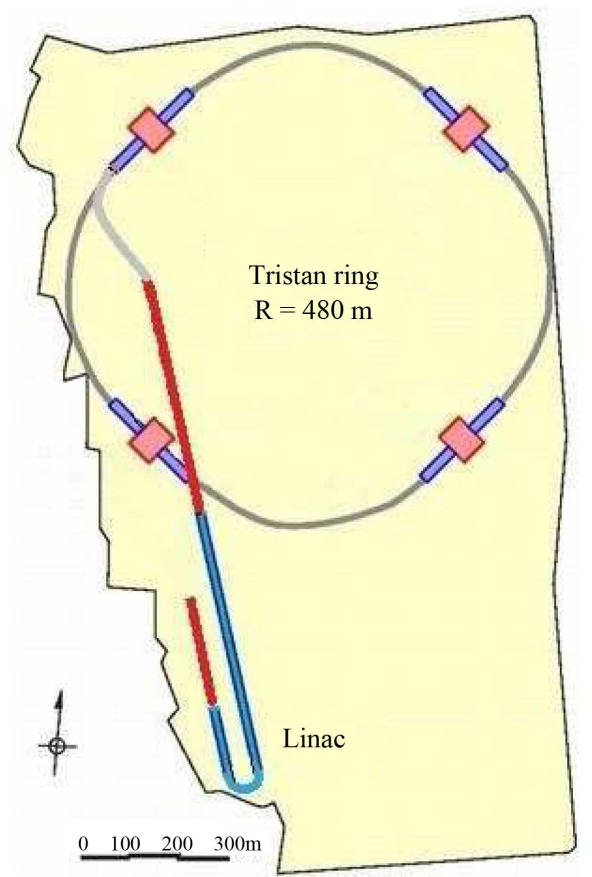

Figure 2. A sketch of the KEK site showing the Tristan ring and the existing electron linac (in blue). The lines drawn in red indicate possible extensions of the present linac tunnels. Alternatively, a new underground linac tunnel could be excavated at a greater depth in case a larger SCL beam energy (up to $25 \mathrm{GeV}$ ) is required.

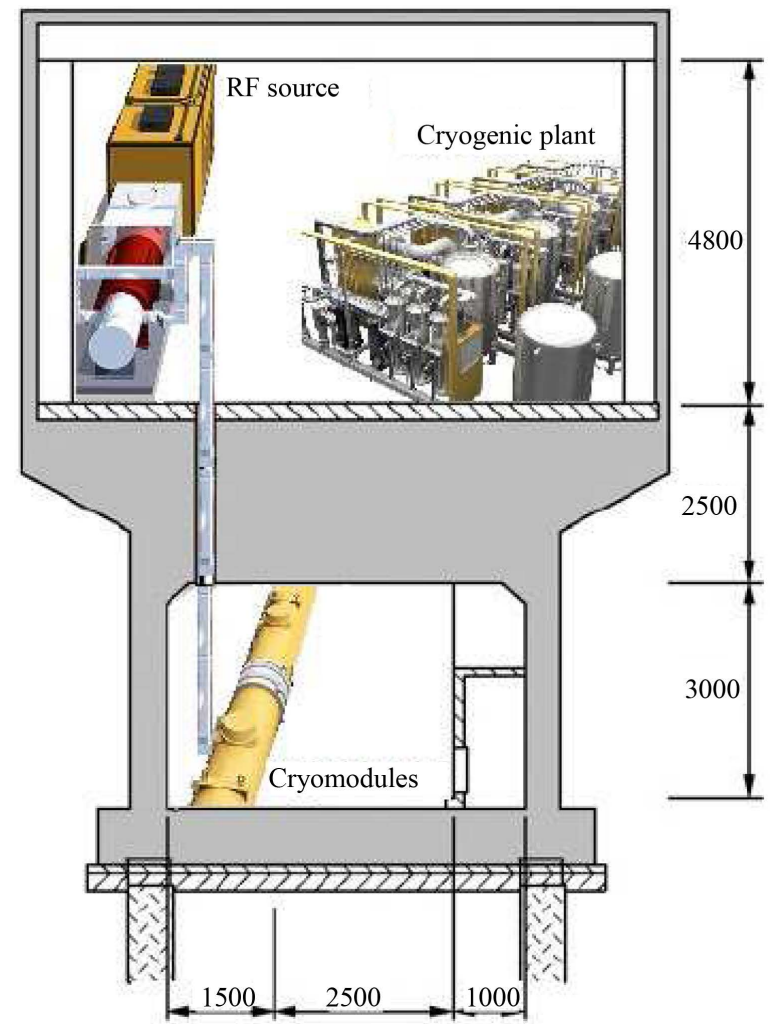

Figure 3. Front view of the linac tunnel and the klystron gallery housing the cryomodules, RF sources and cryogenic plant units of the proposed superconducting linac complex. 
Table 1. Parameters of the SCL.

\begin{tabular}{cc}
\hline Beam energy & $20 \mathrm{GeV}$ \\
Beam power & $15 \mathrm{MW}$ \\
Repetition rate & $20 \mathrm{~Hz}$ \\
Protons per pulse & $2.3 \times 10^{14}$ \\
Beam pulse length & $1.2 \mathrm{~ms}$ \\
Average current per pulse & $31 \mathrm{~mA}$ \\
Duty cycle & $2.4 \%$ \\
RF frequency & $1.3 \mathrm{GHz}$ \\
Klystron average power & $150 \mathrm{KW}$ \\
Klystron peak power & $5 \mathrm{MW}$ \\
Klystron pulse length & $1.5 \mathrm{~ms}$ \\
Effective accelerating gradient & $20 \mathrm{MV} / \mathrm{m}$ \\
Peak power per coupler & $460 \mathrm{~kW}$ \\
\hline
\end{tabular}

type cavities and $I \sim 30 \mathrm{~mA}, \tau_{\mathrm{f}} \approx 0.3 \mathrm{~ms}$. Since $\tau=1.5 \mathrm{~ms}$, the beam pulse length $\tau_{\mathrm{p}} \approx 1.2 \mathrm{~ms}$.

\subsection{Main Characteristics of an ILC-Type Linac}

The main characteristics of a linear accelerator are determined by the properties of its rf source (klystrons) and accelerating cavities. For a pulsed ILC-type superconducting linac, one of the currently available rf sources is the Toshiba E3736 Multi-Beam Klystron [3]. This source has the following well-tested specifications: rf frequency-1.3 GHz; peak rf power-10 MW; average power-150 $\mathrm{kW}$; efficiency-65\%; pulse length $-1.5 \mathrm{~ms}$; repetition rate- $10 \mathrm{~Hz}$. If the repetition rate of the Toshiba klystron is increased by a factor of two, while its peak power is reduced by the same factor (thus keeping the average power constant) one obtains the klystron specifications presented in Table 1. For such a klystron, a suitable $20 \mathrm{~Hz}$ pulse modulator has to be developed.

A very important parameter that determines, to a large extent, the power conversion efficiency of a klystron is its perveance, defined by

$$
K \equiv \frac{I_{0}}{U^{3 / 2}}
$$

In this expression, $I_{0}$ is the beam current and $U$ is the anode voltage. Since there is an upper limit to the applied voltage, low perveance can be only obtained by operating with low currents. For single-beam klystrons, this requirement is not compatible with the need for high output power. With this in mind, multi-beam klystrons (MBK) were originally developed in the 1960s [4]. An MBK is a parallel assembly of low-current (low-perveance) beamlets within a common rf structure, which efficiently generates high output power. Using Equation (3), the output rf power of an MBK can be expressed as

$$
P_{\mathrm{k}}=\eta I_{0} U=\eta K U^{5 / 2}
$$

where $\eta$ is the klystron efficiency and $I_{0}=N_{\mathrm{b}} I_{\mathrm{b}}$ is the total beam current; 
$N_{\mathrm{b}}$ is the number of beamlets and $\boldsymbol{I}_{\mathrm{b}}$ is the current carried by each beamlet. For Toshiba's E3736 MBK, $\eta=65 \%, U=116 \mathrm{kV}$ and $I_{0}=134 \mathrm{~A}$. Hence, $K=3.4 \times 10^{-6} \mathrm{~A} / \mathrm{V}^{3 / 2}$, klystron's peak power $P_{\mathrm{k}}=10 \mathrm{MW}$ and its average power $\bar{P}_{\mathrm{k}} \equiv P_{\mathrm{k}} \times \tau R=150 \mathrm{~kW}$ for $\tau=1.5 \mathrm{~ms}$ and $R=10 \mathrm{~Hz}$. Since the klystron has six beamlets, $I_{\mathrm{b}}=22.3 \mathrm{~A}$.

In order to increase the beam power of the SCL, $R$ could be increased to 20 $\mathrm{Hz}$ (see Equation (1)). As already mentioned, the peak power of the klystron would then have to be reduced to $5 \mathrm{MW}$, so that its average power is still $150 \mathrm{~kW}$ for the klystron pulse length $\tau=1.5 \mathrm{~ms}$ (see Table 1 ).

The basic properties of a $1.3 \mathrm{GHz}$ superconducting ILC-type cavity are presented, e.g., in [5]. There are two important parameters that characterize rf cavities: the accelerating gradient $\mathrm{E}_{\text {acc }}$ and the unloaded quality factor $Q_{0}$. The former is a measure of how much the energy of a particle is increased over a given length of the linac (typically expressed in units of $\mathrm{MV} / \mathrm{m}$ ), while the latter specifies how well the cavity can sustain the stored $\mathrm{rf}$ power. A higher value of $Q_{0}$ implies a lower rate of power loss realtive to the stored energy ${ }^{1}$. ILC-type cavities must have a nominal $Q_{0}$ greater than $1 \times 10^{10}$ (a dimensionless parameter) at $\mathrm{E}_{\text {acc }}=31.5 \mathrm{MV} / \mathrm{m}$.

Each ILC-type cryomodule for the proposed SCL would contain eight niobium 9-cell cavities and a quadrupole magnet at its centre. Other major components of such a cryomodule are the vacuum vessel, thermal and magnetic shields, cryogenic piping, interconnections, etc. The inactive spaces between cavities or cryomodules (the "packing fraction") are responsible for a substantial reduction in the average accelerating gradient of the linac. The beam physics and the lattice design of the superconducting L-band linac described in [6] are applicable to the SCL.

The average usable accelerating gradient in ILC-type cavities is $\overline{\mathrm{E}}_{\mathrm{acc}}=29.3 \pm 5.1 \mathrm{MV} / \mathrm{m}$ [7]. Taking into account an estimated linac "packing fraction" of about $70 \%$, the effective accelerating gradient of the SCL is $\mathrm{E}_{\text {eff }} \approx 20 \mathrm{MV} / \mathrm{m}$. Hence, the total length of a $20 \mathrm{GeV}$ linac is $\sim 1000 \mathrm{~m}$.

Since the length of an ILC 9-cell cavity is $1 \mathrm{~m}$, a linac with $E_{b}=20 \mathrm{GeV}$ would require about $N_{\text {cav }}=(20000 \mathrm{MeV}) /(29 \mathrm{MeV})=690$ cavities. The average input rf power per cavity is thus $\bar{P}_{\text {cav }}=P_{\mathrm{b}} / N_{\text {cav }} \approx 22 \mathrm{~kW}$, and the corresponding peak power $P_{\text {cav }} \equiv \bar{P}_{\text {cav }} / D=916 \mathrm{~kW}$. Although this value is acceptable for a pulsed linac with $D \sim 2 \%$, it would be prudent to use two rf couplers per cavity. In that case the peak rf power per coupler would be about $460 \mathrm{~kW}$.

For $\mathrm{E}_{\mathrm{acc}}=30 \mathrm{MV} / \mathrm{m}$, ohmic losses in an ILC 9-cell cavity amount to $P_{\mathrm{c}}=100 \mathrm{~W}$ in the CW mode of operation, but only $P_{\mathrm{c}}=(100 \times D) \mathrm{W}=2.4 \mathrm{~W}$ (plus static loss) in the pulsed mode with a duty factor $D=0.024$. Because of large ohmic losses, which scale with the square of the accelerating gradient, $\mathrm{E}_{\text {acc }}$ is generally lower in the $\mathrm{CW}$ mode of operation than in the pulsed mode.

${ }^{1}$ The $Q$ factor of an $\mathrm{rf}$ cavity is defined as $Q=2 \pi \times$ (energy stored/energy dissipated per cycle). For large values of $Q$, the $Q$ factor is approximately the number of oscillations required for the energy of a freely oscillating system to fall off to $\mathrm{e}^{-2 \pi}$, or $0.2 \%$, of its original value. 


\subsection{Proton Injector (PI)}

A typical $\sim 1 \mathrm{GeV}$ proton linear accelerator consists of three main sections:

- Front end, comprising a proton source and a radiofrequency quadrupole accelerator;

- Medium-velocity linac, which accelerates proton beams to 100 MeV;

- High-velocity linac, which accelerates protons to energies exceeding $1 \mathrm{GeV}$.

The most complex part of a proton linac is the low-energy (low- $\beta$ ) section, situated between the proton (or ion) source and the first drift-tube-based accelerating section. The continuous beam of protons coming from an electron cyclotron resonance (ECR) source [8] has to be focused, bunched and accelerated in the first $\mathrm{rf}$ structure. These three essential functions are nowadays successfully performed by radio frequency quadrupoles (RFQ) [9]. However, the beam has to be shrunk before it can be fed into an RFQ. This is accomplished within a low-energy beam transport (LEBT) section by means of cylindrical magnets (solenoids).

As soon as the beam is bunched---which is essential for further accelerationit enters a medium-energy beam transport (MEBT) section, where it is collimated and steered from the RFQ into the medium-velocity linac (MVL). The MEBT may also contain a number of buncher cavities. Inside the MVL, the beam is accelerated to about $100 \mathrm{MeV}$ ( $\beta \sim 0.1$ to 0.5$)$. The MVL usually contains normal-conducting drift-tube linac (DTL) and cell-coupled drift tube linac (CCDTL) structures. A DTL incorporates accelerating components of increasing length in order to match precisely the increase in beam velocity, while quadrupole magnets provide strong focusing. The main advantage of using CCDTL structures is that they provide longitudinal field stability.

High-velocity linac (HVL) structures accelerate the beam to energies around 1 $\mathrm{GeV}$. They consist either of normal-conducting side-coupled linac (SCL) structures $^{2}$ or superconducting elliptical cavities. The latter offer some advantages over the former, such as higher accelerating gradients and lower operating costs. The superconducting HVL can also feature spoke resonators, characterized by their simplicity, high mechanical stability and compact size [10].

The European Spallation Source (ESS) is an example of a typical $\sim 1 \mathrm{GeV}$ proton linear accelerator [11] (see Figure 4). The transverse beam size along the linac varies in the range $1-4 \mathrm{~mm}$, and the bunch length decreases from $1.2 \mathrm{~cm}$ to $3 \mathrm{~mm}$ towards the end of the linac.

One of the main concerns in the design of a high-power proton linac is to restrict beam losses. A careful beam dynamics study is therefore needed in order to avoid halo formation, a major source of beam loss. Another important issue is the preservation of beam emittance [6].

High-power proton linear accelerators have a wide range of applications including spallation neutron sources, nuclear waste transmutation, production of radioisotopes for medical use, etc. A number of laboratories worldwide have ex-

${ }^{2}$ The main reason for using these $\pi / 2$-mode structures is that long chains of coupled cavities are often required for an efficient use of high-power rf sources [10]. 


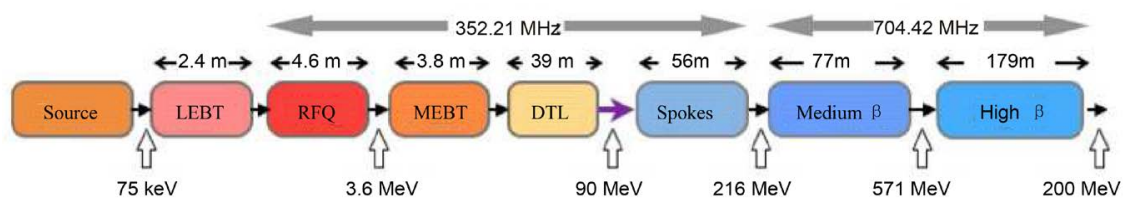

Figure 4. Block diagram of the ESS linac [11]. The RFQ and DTL structures are normalconducting, while the spoke resonator and elliptical cavities are superconducting.

pressed interest in building "proton drivers" that would primarily deliver highintensity neutrino, kaon and muon beams [12] [13].

\section{Physics at the Proposed Facility}

The physics potential of a multi-MW "proton driver" is extensively discussed, for instance, in [14]. This note is mainly concerned with the application of a high-intensity proton source to investigate the properties of long-baseline neutrino oscillations. A unique feature of the proposed facility is the use of a superconducting linac to accelerate both protons and electrons, which considerably increases its physics potential. As described in [15] [16], polarized electron and positron beams can be used to study the structure of composite particles and the dynamics of strong interactions, as well as to search for new physics beyond the Standard Model.

\subsection{Neutrino Flavor Oscillations and Leptonic CP Violation}

The observed transformation of one neutrino flavor into another ("neutrino oscillation") indicates that these particles have finite masses. Cosmological data suggest that the combined mass of all three neutrino species is a million times smaller than that of the next-lightest particle, the electron [17]. The phenomenon of neutrino oscillations implies not only the existence of neutrino mass, but also of neutrino mixing. That is, the neutrinos of definite flavor are not particles of definite mass (mass eigenstates), but coherent quantum-mechanical superpositions of such states. Converesely, each neutrino of definite mass is a superposition of neutrinos of definite flavor. Neutrino mixing is large, in striking contrast to quark mixing. Whatever the origin of the observed neutrino masses and mixings, it implies a profound modification of the Standard Model.

Mathematically, the phenomenon of neutrino mixing can be expressed as a unitary transformation relating the flavor and mass eigenstates. The neutrino oscillation rate depends, in part, on (1) the difference between neutrino masses and (2) the three parameters in the transformation matrix known as mixing angles. The complex phase factors in the transformation matrix (also called mixing matrix) are associated with the violation of CP symmetry in the lepton sector. The size of the CP violation is determined both by the phases and the mixing angles.

Experiments with high-intensity neutrino beams are designed primarily to explore the mass spectrum of the neutrinos and their properties under the $\mathrm{CP}$ symmetry, and thus provide a deeper insight into the nature of these elusive par- 
ticles and their role in the universe. For instance, if there is experimental evidence for $\mathrm{CP}$ violation in neutrino oscillations, it could be used to explain the observed asymmetry between matter and antimatter [18].

To search for $\mathrm{CP}$ violation in neutrino oscillations, a 100 kiloton water Cherenkov detector could be built at Okinoshima, located at a distance

$\mathrm{L}_{2} \approx 650 \mathrm{~km}$ from KEK. Using the proposed KEK "proton driver", the detector at Okinoshima and the existing 0.022 Mt Super-Kamiokande detector (situated at a distance $\mathrm{L}_{1} \approx 300 \mathrm{~km}$ from KEK), the neutrino mass hierarchy could be determined either by comparing the $v_{e}$ appearance probabilities measured at the two vastly different baseline lengths $\mathrm{L}_{1}$ and $\mathrm{L}_{2}$, or by measuring at $\mathrm{L}_{1}$ and $\mathrm{L}_{2}$ the neutrino energy of the first oscillation maximum. Once the mass hierarchy is determined, the $\mathrm{CP}$-violating phase in the mixing matrix can be measured with a precision of $\pm 20^{\circ}$, assuming that $2.5 \times 10^{21}$ protons are delivered on target for both $\nu_{e}$ and $\overline{\nu_{e}}$ beams [19].

\section{Proton Target and Magnetic Horn}

The main challenge in the design of a multi-MW neutrino beam facility is to build a proton target that could dissipate large amounts of deposited energy, withstand the strong pressure waves created by short beam pulses, and survive long-term effects of radiation damage. Simulation studies of the pion production and energy deposition in different targets (liquid mercury jet, tungsten powder jet, solid tungsten bars and gallium liquid jet) are presented in [20]. Those studies also provide estimates of the amount of concrete shielding needed to protect the environment from the high radiation generated by each target. A proof-ofprinciple demonstration of a $4 \mathrm{MW}$ target station comprising a liquid mercury jet inside a $20 \mathrm{~T}$ solenoidal magnetic field is described in [21]. Alternatively, one could use a rotating, gas-cooled tungsten target that would require the least amount of development effort, and would also have good thermal and mechanical properties [11]. A $15 \mathrm{MW}$ proton beam could be separated by a series of magnets into four beam lines. Each of the four beams would be focused by a series of quadrupoles and correctors to an assembly consisting of four targets and the same number of magnetic horns (see, e.g., [22]).

To maximize the discovery potential of a neutrino beam facility, it is important to properly design the magnetic horn that focuses the charged particles produced in the proton target. For proton beam pulses lasting $1 \mathrm{~ms}$, a DC horn has been designed at KEK by Yukihide Kamiya [23]. The toroidal magnetic field of the horn, characterized by $\mathrm{B}(r)=$ const., is generated by hollow aluminium conductors containing water. The strength of the magnetic field $\mathrm{B}=0.2 \mathrm{~T}$, and its length $\ell=5 \mathrm{~m}$; hence, $\mathrm{B} \cdot \ell=1 \mathrm{~T} \cdot \mathrm{m}$. The radius of the magnet, $r$, is determined by $r=L \tan (\theta)+\ell \tan (\theta / 2)$, where $\theta \approx 0.03+0.3 / p$ is the initial angle a charged pion makes with respect to the proton beam direction, $L$ is the distance from the target to the horn, and $p$ is the pion momentum. For example, if $L=5 \mathrm{~m}$ then $r \approx 5 \mathrm{~m}$. The total power generated in the conductors is about $10 \mathrm{MW}$. 


\subsection{Physics with Polarized Electrons and Positrons}

Electron and positron beams, polarized and/or unpolarized, can be used to study the structure of composite particles and the dynamics of strong interactions, as well as to search for new physics beyond the Standard Model. A detailed description of the physics potential of a facility that can provide such beams (e.g., the upgraded CEBAF facility at Jefferson Lab or the proposed KEK superconducting linac) is presented in [15] [16].

Polarized positrons are created in a conversion target by circularly polarized photons, which themselves are produced when polarized laser light is Comptonbackscattered on a high-energy electron beam [24]. Circularly polarized photons can also be produced by bremsstrahlung from polarized electrons [25]. Using polarized electrons and positrons, the nucleon electromagnetic form factors and generalized parton distributions can be determined in a model-independent way [16].

Among the physics topics discussed in [15], parity violation in electron-electron (Mø ller) scattering is of particular interest. Mø ller scattering is a purely leptonic process that allows high-precision tests of the Standard Model. At four-momentum transfers much smaller than the mass of the $\mathrm{Z}$ boson ( $q^{2} \ll m_{Z}^{2}$ ), the parity-violating asymmetry, $A$, is dominated by the interference between the electromagnetic and neutral weak amplitudes [26]. By definition,

$$
A \equiv \frac{\mathrm{d} \sigma_{R}-\mathrm{d} \sigma_{L}}{\mathrm{~d} \sigma_{R}+\mathrm{d} \sigma_{L}} \approx \frac{f_{\mathrm{Z}}^{R}-f_{Z}^{L}}{f_{\gamma}}
$$

In this expression, $\mathrm{d} \sigma_{R}\left(\mathrm{~d} \sigma_{L}\right)$ is the differential cross-section for right-handed (left-handed) electron scattering on an unpolarized target:

$$
\mathrm{d} \sigma_{R, L} \propto\left|f_{\gamma}+f_{Z}^{R, L}\right|^{2} \approx\left|f_{\gamma}\right|^{2}+2 f_{\gamma} f_{Z}^{R, L}
$$

where $f_{\gamma}$ and $f_{Z}^{R, L}$ are the scattering amplitudes with $\gamma$ and $Z$ exchange, respectively. From the four Feynman diagrams in Figure 6 of [27], one can readily obtain the Born amplitudes for Mø ller scattering mediated by photons and $\mathrm{Z}$ bosons. The weak neutral current amplitudes are functions of the weak mixing (or Wienberg) angle $\theta_{\mathrm{w}}$, which relates the weak coupling constants $g_{\mathrm{w}}$ and $g_{z}$ to the electromagnetic coupling constant. As shown in [27], the polarization asymmetry for polarized electron scattering on an unpolarized target is given by

$$
A^{\mathrm{Born}}=m_{e} \mathrm{E} \frac{G_{\mathrm{F}} Q_{\mathrm{w}}^{e}}{\sqrt{2} \pi \alpha} F(\theta)
$$

where $m_{e}$ is the mass of the electron, $\mathrm{E}$ is the incident beam energy, $G_{\mathrm{F}}$ is the Fermi coupling constant characterizing the strength of the weak interaction, $\alpha$ is the fine structure constant, and $F(\theta)$ is a function of the scattering angle in the center-of-mass frame. The weak charge of the electron, $Q_{\mathrm{w}}^{e}=1-4 \sin ^{2} \theta_{\mathrm{w}}$, is proportional to the product of the electron's vector and axial-vector couplings to the $\mathrm{Z}$ boson.

Since the value of $\sin ^{2} \theta_{\mathrm{w}}$ is close to $1 / 4$, there is an enhanced sensitivity of $A$ 
to small changes in the Weinberg angle. The value of $\theta_{\mathrm{w}}$ varies as a function of the four-momentum transfer, $q$, at which it is measured. This variation, or "running", is a key prediction of the Standard Model. The one-loop electroweak radiative corrections to $A^{\text {Born }}$ (calculated once the renormalized parameters in (7) are properly defined) reduce its Born value by $\sim 40 \%$. This effect can be attributed to an increase of $\sin ^{2} \theta_{\mathrm{w}}\left(q^{2}\right)$ by $3 \%$ as the four-momentum transfer "runs" from $q^{2}=m_{Z}^{2}$ to $q^{2} \approx 0$ [28]. The precision with which $\sin ^{2} \theta_{\mathrm{w}}$ can be measured in the MOLLER experiment [29] (where a $11 \mathrm{GeV}$ longitudinally polarized electron beam scatters on atomic electrons in a liquid hydrogen target) is shown in Figure 5.2 of [15].

Many of the electroweak meaurements obtained so far may be combined to provide a global test of consistency with the SM. Since the Higgs-boson mass affects the values of electroweak observables through radiative corrections, it is of fundamental importance to test the agreement between the directly measured value of $m_{H}$ and that inferred from the measurements of electroweak parameters $m_{W}, m_{\text {top }}$ and $\sin ^{2} \theta_{\mathrm{w}}$ (see Figure 5.2 in [15]). High-precision electroweak measurements, therefore, represent a natural complement to direct studies of the Higgs sector.

Apart from providing a comprehensive test of the SM, precision measurements of weak neutral current interactions at $q^{2} \ll m_{Z}^{2}$ also allow indirect access to new physics phenomena beyond the $\mathrm{TeV}$ energy scale. For instance, such measurements can be used to look for hypothetical Z' bosons, 4-fermion contact interactions, or very weekly coupled low-mass "dark bosons" [30].

\subsection{Rare Kaon Decays}

$\mathrm{CP}$ violation was introduced in the SM by increasing the number of quark and lepton families to at least three (M. Kobayashi and T. Maskawa, 1973). This idea became very attractive with the subsequent discovery (in 1977) of the bottom quark, which forms, together with the top quark (discovered in 1995), a third family of quarks. It is a remarkable property of the Kobayashi-Maskawa model that quark mixing and CP violation are intimately related [31].

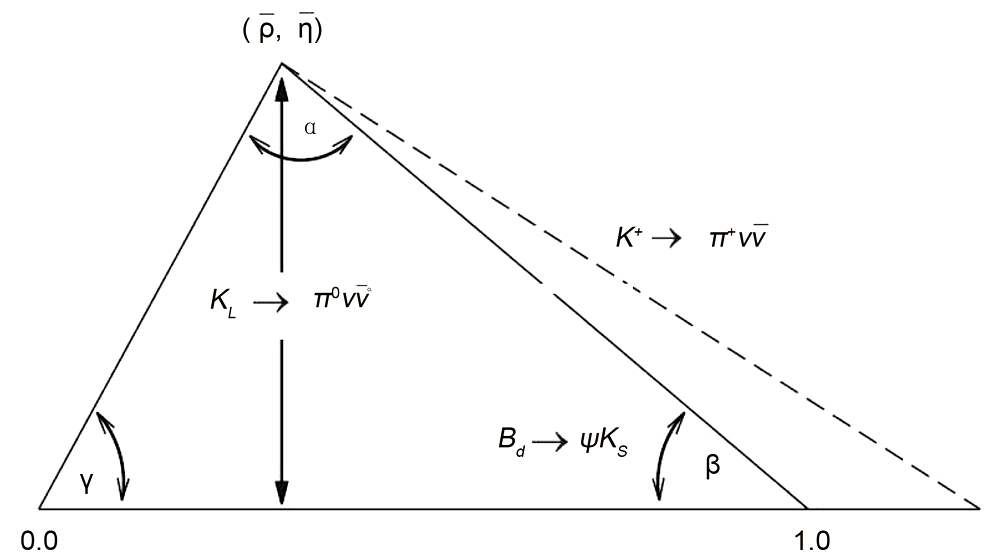

Figure 5. Unitarity triangle from $K \rightarrow \pi v \bar{v}$ decays. The displacement of the bottomright vertex is due to the charm-quark contribution to $K^{+} \rightarrow \pi^{+} \nu \bar{v}$. 
A deeper insight into CP violation is expected to be gained from precision measurements of rare kaon decays such as $K_{\mathrm{L}}^{0} \rightarrow \pi^{0} v \bar{\nu}$ and $K^{+} \rightarrow \pi^{+} v \bar{v}$. Both decays are theoretically 'clean' because hadronic transition amplitudes are matrix elements of quark currents between mesonic states, which can be extracted from the leading semileptonic decays using isospin symmetry. Since photons do not couple to neutrinos, $K \rightarrow \pi v \bar{v}$ decays are entirely due to second-order weak processes determined by Z-penguin and $\mathrm{W}$-box diagrams [31].

The process $K_{\mathrm{L}}^{0} \rightarrow \pi^{0} v \bar{v}$ proceeds almost entirely through direct CP violation, and is completely determined by "short-distance" one-loop diagrams with top quark exchange. The Standard Model predicts its branching ratio to be [32]

$$
B\left(K_{\mathrm{L}}^{0} \rightarrow \pi^{0} v \bar{v}\right)=(2.43 \pm 0.39) \times 10^{-11} \quad \text { theoretical value }
$$

This decay is an important source of information on higher-order effects in electroweak interactions, and thus can serve as a probe of new physics (see [33] and references therein).

The decay $K^{+} \rightarrow \pi^{+} v \bar{\nu}$ receives both CP-conserving and CP-violating contributions. It has theoretical uncertainties that are somewhat larger than those in the process $K_{\mathrm{L}}^{0} \rightarrow \pi^{0} v \bar{v}$. Since both decays involve one-loop Feynman diagrams with top quark exchange, they can yield valuable measurements of the CKM matrix elements $\left|V_{t d}\right|$ and $\left|V_{t s}\right|$. The quantity $\operatorname{Im} V_{t s}^{*} V_{t d}$, which can be obtained from $K_{\mathrm{L}}^{0} \rightarrow \pi^{0} v \bar{\nu}$ alone, plays a central role in the phenomenology of CP violation in $\mathrm{K}$ decays; this quantity is related to the Jarlskog parameter, the invariant measure of CP violation in the Standard model [31] [34].

By measuring the branching ratios of both $K \rightarrow \pi \nu \bar{v}$ decay modes, the unitarity triangle of the CKM matrix can be completely determined (see Figure 5), provided the matrix element $V_{c b}$ and the top quark mass are known [34]. Of particular interest is the unitarity triangle parameter $\sin 2 \beta$, which can also be determined from the decay $B_{d} \rightarrow \Psi \mathrm{K}_{s}$. Both determinations of this parameter have to coincide if the Standard Model is valid [33].

The current branching ratio measurement of the charged decay mode,

$$
B\left(K^{+} \rightarrow \pi^{+} v \bar{v}\right)=\left(17.3_{-10.5}^{+11.5}\right) \times 10^{-11} \text { measured value }
$$

is based on the seven candidate events observed by the experiment E787/E949 at Brookhaven [35]. This result is consistent with $(7.8 \pm 0.80) \times 10^{-11}$, the value predicted by the SM [32].

As shown in Figure 11 of [33], the kaon yield rises rapidly as a function of the incident proton momentum. From the figure one infers that the minimum energy of the proton beam should be about $20 \mathrm{GeV}$, for otherwise the kaon yield would be severely reduced. At the proposed KEK facility, the total proton beam energy would be $(2.5+20) \mathrm{GeV}$. A $70 \mathrm{GeV}$ proton synchrotron could be installed, at a later stage, inside the Tristan ring in order to increase the proton beam energy -albeit at the cost of a considerably lower beam power. For a given kaon yield, the required beam power would be lowest at $\mathrm{E}_{\mathrm{b}}=30-100 \mathrm{GeV}$ [33]. 


\subsection{A Novel $g$-2 Experiment with Ultra-Slow Muons}

A charged elementary fermion has a magnetic dipole moment $\mu=g_{s}(q / 2 m) s$ aligned with its spin $s$. The proportionality constant $g_{s}$ is the Landé $g$-factor, $q$ is the charge of the particle and $m$ is its mass. Dirac's theory of the electron predicts that $g_{s}=2$. For the electron (e), muon $(\mu)$ and tau lepton $(\tau)$, this prediction differs from the observed value by a small fraction of a percent. The difference is the anomalous magnetic moment, the anomaly is defined by $a \equiv\left(g_{s}-2\right) / 2 \sim 10^{-3}$. In the Standard Model, three distinct classes of Feynman diagrams contribute to the value of the anomaly for each lepton species: (1) the dominant QED terms that contain only leptons and photons; (2) terms that involve hadrons; and (3) electroweak terms containing the Higgs, $\mathrm{W}$ and $\mathrm{Z}$ bosons. The muon anomaly $a_{\mu}$ is about $\left(m_{\mu} / m_{e}\right)^{2} \sim 43000$ times more sensitive to the existence of yet unknown heavy particles than the electron anomaly $a_{e}$. The value of $a_{\mu}\left(a_{e}\right)$ is sensitive to new physics at the scale of a few hundred $\mathrm{GeV}(\mathrm{MeV})$ [36].

The current experimental uncertainty on $a_{\mu}$ is $\pm 0.54 \mathrm{ppm}$. In a novel $g$ - 2 experiment [37], the main aim of which is to reduce this uncertainty to \pm 0.1 ppm, $3 \mathrm{GeV}$ protons impinge on a graphite target and produce pions that are stopped in the target. Some of the positive pions are brought to rest near the surface of the target, where they decay into positive muons with momenta $p_{\mu}=30 \mathrm{MeV} / \mathrm{c}$ and $100 \%$ spin polarization. The muons are collected using a large-aperture solenoid and transported to a silica-aerogel target in which they form muonium (electron $-\mu^{+}$) atoms. As the atoms slowly diffuse from the target, they are ionized by a pulsed laser to produce $50 \%$ polarized muons with very low momenta ${ }^{3}$. Those "ultra-slow" muons $\left(4 \times 10^{4} /\right.$ pulse $)$ are then accelerated to $p_{\mu}=300 \mathrm{MeV} / \mathrm{c}$ by two linacs, and injected into a magnetic storage ring that contains a $3 \mathrm{~T}$ solenoid with a diameter of $66 \mathrm{~cm}$. After injection, the muons circulate orthogonal to the magnetic field $\mathbf{B}$. An orbiting muon decays within $6.6 \mu$ s into a positron, a neutrino and an antineutrino:

$\mu^{+} \rightarrow \mathrm{e}^{+}+v_{e}+\bar{v}_{\mu}$. The experiment is also designed to measure the electric dipole moment $d_{\mu}=\eta(q \hbar / 2 m) s$ of the muon (see below).

The highest-energy positrons, preferentially emitted parallel to the muon spin direction in the $\mu^{+}$rest frame, are Lorentz-boosted to become the highest-energy positrons in the lab frame. Hence, the angular distribution of those positrons has its maximum in the direction of the muon spin [17]. By measuring the energy and time distributions of positrons one can determine the average spin direction. The time spectrum will show the muon lifetime modulated by the spin precession frequency. The relative precession of the spin with respect to the direction of the particle velocity $u$ is given by

$$
\omega_{a}+\omega_{\eta} \propto a_{\mu} \mathbf{B}-(\eta / 2)(\beta \times \mathbf{B})
$$

where $\omega_{a}$ and $\omega_{\eta}$ arise from $a_{\mu}$ and $d_{\mu}$, respectively, and $\beta \equiv u / c$. ${ }^{3} \mathrm{~A}$ much higher level of polarization can be obtained by using a magnetic field to align the particle spins [37]. 
Since the rotation axes due to $a_{\mu}$ and $d_{\mu}$ are orthogonal, the corresponding signals can be separated [37]. In the case of $\mu_{\mu}$, the anomalous precession period is $2.2 \mu \mathrm{s}$, about 300 times the cyclotron period. Assuming that muons are $100 \%$ polarized, $1.5 \times 10^{12}$ positrons have to be detected for a measurement precision of $0.1 \mathrm{ppm}$ [37].

\section{An XFEL Based on the Proposed Superconducting Linac}

To record the dynamics of atoms requires a probe with Ångstrom $\left(10^{-10} \mathrm{~m}\right)$ wavelength and femtosecond temporal duration $\left(10^{-15} \mathrm{~s}\right)$. Such probes have recently become available with the advent of $X$-ray free-electron lasers (XFELs). The ultrashort pulse duration of an XFEL matches the timescale of non-equilibrium microscopic processes such as electron transfer in molecules, evolution of chemical reactions, vibration dynamics in solid state systems, etc. Optical lasers are also capable of producing pulses of femtosecond duration, but lack the required spatial resolution.

The spectral brightness (or brilliance), $B$, of a radiation field can be expressed, in practical units, as the number of photons per second passing through a given cross-sectional area and within a given solid angle and spectral bandwidth (BW). This quantity determines how much monochromatic radiation can be focused onto a tiny spot on the target. The peak spectral brightness-the brightness measured during the very short duration of an FEL pulse-of the two presently most powerful XFEL facilities (LCLS at SLAC in the United States and SACLA at SPring-8 in Japan) is billion times higher than that of any synchrotron radiation source.

Owing to the high intensity of XFEL radiation, laser-irradiated atoms, molecules and atomic clusters can be excited into previously unknown states. Although high-intensity pulses may also destroy molecular structures, they can still be used to produce high-resolution X-ray diffraction patterns, from which real-space images of the atomic positions in molecules can be reconstructed. In a typical "pump-probe" experiment, the evolution of a chemical (or biochemical) reaction, initiated by an optical or IR laser pulse, is observed by a time-delayed $\mathrm{X}$-ray pulse. By varying the delay, such stroboscopic measurements result in femtosecond "movies" of the evolving system. Note that, due to their long pulse duration, X-rays from synchrotron light sources can be used to image atomic structures only in static measurements.

Nanometre-scale molecular imaging is made possible by the high degree of coherence of the XFEL radiation. The coherence quality of a light source is best described by the degeneracy parameter $D$, defined as the number of emitted photons per coherent phase-space volume per coherence time:

$$
D \equiv \frac{B \lambda^{3}}{4 c} \approx 8.3 \times 10^{-25} B \lambda^{3}
$$

Here $B$ is the brilliance and $\lambda[0.1 \mathrm{~nm}]$ the wavelength of the source.

Free-electron lasing is achieved by a single-pass, high-gain FEL amplifier op- 
erating in the so-called self-amplified spontaneous emission (SASE) mode. An FEL consists of an electron linear accelerator and an undulator, a long periodic array of magnets with period $\lambda_{u}$. The wavelength of the first harmonic of the observed FEL radiation is given by [38] [39]

$$
\lambda=\frac{\lambda_{u}}{2 \gamma^{2}}\left(1+\frac{K^{2}}{2}+\gamma^{2} \theta^{2}\right)
$$

where the dimensionless quantity

$$
K=\frac{e \mathrm{~B}_{0} \lambda_{u}}{2 \pi m_{e} c}=0.934 \mathrm{~B}_{0}[\text { Tesla }] \lambda_{u}[\mathrm{~cm}]
$$

is the undulator deflection parameter and $\gamma$ is the Lorentz factor of the electron beam. Typically, $\lambda_{u} \approx 3 \mathrm{~cm}$ and $\gamma \approx 10^{4}$; hence, $\lambda \approx 0.1 \mathrm{~nm}$.

The emission of radiation in an undulator does not occur at one wavelength, but in a wavelength band of width $\Delta \lambda$ around the central value given by Equation (12). Each electron propagating through the undulator emits a wave train consisting of a number of wavelengths equal to the number of undulator periods, $N_{u}$. The time duration $\Delta t$ of this pulse is the pulse length $L_{p} \equiv N_{u} \lambda$ divided by the speed of light: $\Delta t=N_{u} \lambda / c$. A pulse of duration $\Delta t$ has a frequency bandwidth $\Delta v \sim 1 / \Delta t$. Hence, $\Delta v \sim c / N_{u} \lambda=v / N_{u}$, because $\lambda=c / v$. Thus,

$$
\frac{\Delta v}{v}=\frac{\Delta \lambda}{\lambda} \sim \frac{1}{N_{u}} \approx 10^{-3}
$$

The wave train is not monochromatic due to its finite length. For typical values $N_{u} \approx 10^{3}$ and $\lambda \approx 0.1 \mathrm{~nm}$, one obtains $\Delta t \approx 0.33 \mathrm{fs}$. Since the electrons are distributed throughout a bunch, the pulse duration is increased to $\tau_{p} \sim\left(\sigma_{z} / L_{p}\right) \Delta t \approx 80 \mathrm{fs}$, where $\sigma_{z} \approx 25 \mu \mathrm{m}$ is the bunch length and $L_{p} \approx 0.1 \mu \mathrm{m}$. An $80 \mathrm{fs}$ pulse, therefore, consists of many micropulses of $0.33 \mathrm{fs}$ duration.

The 'shot noise' in an electron beam, the origin of which is the random emission of the electrons from a photocathode, causes random fluctuations of the beam density. The radiation produced by such a beam has amplitudes and phases that are random in both space and time. For this reason, SASE X-ray FELs lack longitudinal (or temporal) coherence, characterized by the coherence length $L_{\text {coh }} \equiv \lambda^{2} / \Delta \lambda \approx 0.1 \mu \mathrm{m}$. This quantity is defined as the distance of propagation over which radiation with spectral width $\Delta \lambda$ becomes $180^{\circ}$ out of phase. The coherence time, defined by $t_{\text {coh }} \equiv L_{\text {coh }} / c \sim 1 / \Delta v$, is much shorter than the pulse duration: $t_{\text {coh }} \approx 0.3 \mathrm{fs}$.

In order to increase the coherence length in the hard X-ray regime (photons with $0.1 \mathrm{~nm}$ wavelength), a "self-seeding" method was tested at LCLS [40]. FEL pulses, generated in the first modular section of the LCLS undulator, are spectrally "purified" by a crystal filter (a diamond monochromator). Since a typical monochromator delays X-rays, the electron bunches exiting the first modular section are appropriately delayed after being diverted around the crystal by a 
compact magnetic chicane (see Figure 1 in [40]). The crystal selects a very narrow part of the spectrum, which is further amplified in the second undulator section where the FEL radiation reaches saturation. At LCLS, "self-seeding" generated X-ray pulses with $\Delta v=0.4-0.5 \mathrm{eV}$ at $v=8-9 \mathrm{keV}$, which represents a factor of $40-50$ bandwidth reduction with respect to SASE [40].

\section{The European XFEL as a Prototype for the Proposed X-Ray FEL}

The European XFEL, currently under construction at DESY (Germany), could serve as a prototype for the proposed X-Ray FEL at KEK. The XFEL at DESY is a free-electron laser based on self-amplified spontaneous emission (SASE) in the $\mathrm{X}$-ray regime. The FEL consists of a $17.5 \mathrm{GeV}$ superconducting electron linear accelerator and a set of undulators that can generate both SASE FEL X-rays and incoherent radiation. A schematic layout of the European XFEL is shown in [41]. Electron bunches, each with a charge of $1 \mathrm{nC}$, are extracted from a photocathode by short ultraviolet laser pulses and then focused and accelerated inside a radio-frequency cavity ("RF gun") to an energy of $120 \mathrm{MeV}$. In order to produce $5 \mathrm{kA}$ peak currents necessary for lasing, the bunches are further accelerated and longitudinally compressed down to $25 \mu \mathrm{m}$ using two magnetic chicanes (at 0.5 and $2.0 \mathrm{GeV}$ ). After traversing the main linac, where their energy is increased to $17.5 \mathrm{GeV}$, the bunches are sent through a number of FEL undulators.

A superconducting linac may accelerate 10 "bunch trains" per second, each train consisting of up to 2700 electron bunches. This results in 27,000 ultrashort $\mathrm{X}$-ray flashes per second. The higher the number of electron bunches, the more scientific instruments can be operated simultaneously. The European XFEL facility will generate ultra-short pulses ( $\leq 100 \mathrm{fs}$ ) of spatially and temporally coherent X-rays with wavelengths in the range $\sim 0.1-5 \mathrm{~nm}$; its peak brilliance is expected to be

$$
B \approx 5 \times 10^{33} \text { photons } / \text { second } / \mathrm{mm}^{2} / \mathrm{mrad}^{2} / 0.1 \% \text { BW }
$$

The coherent superposition of the radiation fields from all microbunches within an electron bunch in the linac is responsible for the nearly monochromatic spectrum and small divergence of the radiation emitted in the forward direction [38]. Recall also that "self-seeding" can substantially improve longitudinal (temporal) coherence of SASE XFEL radiation. Thus, the radiation from an $\mathrm{X}$-ray FEL has a narrow bandwidth, is transversely and longitudinally coherent, and is fully polarized. The coherently emitted XFEL spectral lines appear in addition to the spontaneously emitted undulator spectrum that extends into the $\mathrm{MeV}$ energy region (see, e.g., [42]).

The micropulses that form an FEL pulse give rise to "spikes" in the spectrum. The amplitudes of the micropulses vary greatly as a consequence of the amplified stochastic variations in the electron density. Within a micropulse, the radiation is both transversely and longitudinally coherent. The duration of a micropulse is roughly $t_{\text {coh }}$, the coherence time. In the SASE 1 and SASE 2 undulators at the European XFEL, $t_{\text {coh }}=0.2-0.38 \mathrm{fs}$ [41]. The number of "spikes" in a 
pulse is given by the ratio of the bunch length to the coherence length: $\sigma_{z} / L_{\text {coh }}=(25 \mu \mathrm{m}) /(0.1 \mu \mathrm{m}) \approx 250$.

The spectrum of undulator radiation is sharply peaked around odd harmonics $^{4}$ (see, e.g., [42]). The photon energy that corresponds to the $n^{\text {th }}$ harmonic is given by

$$
\mathrm{E}_{n}[\mathrm{keV}]=0.9496 \frac{n \mathrm{E}_{e}^{2}[\mathrm{GeV}]}{\lambda_{u}[\mathrm{~cm}]\left(1+K^{2} / 2+\gamma^{2} \theta^{2}\right)}
$$

where $\mathrm{E}_{e}$ is the electron beam energy and $\theta$ is the radiation detection angle (with respect to the forward direction). For $\theta=0$ and the SASE 2 undulator parameters $\lambda_{u}=4.8 \mathrm{~cm}$ and $K=6.1$, for example, Equation (16) yields $\mathrm{E}_{1}=12.2 \mathrm{keV} \quad[41]$.

At the exit of the SASE 1 undulator, the photon beam divergence is $\sigma_{\theta} \sim 1 / \gamma \sqrt{N_{u}} \approx 1 \mu \mathrm{rad}$ [38] and the beam size is $70 \mu \mathrm{m} \times 70 \mu \mathrm{m}$, the diameter of a fine needle. This beam can be focused to an area of $0.1 \mu \mathrm{m} \times 0.1 \mu \mathrm{m}$ (the size of a virus) at an experimental station located a couple of hundred meters from the undulator exit [43]. Through variable focusing, the flux density of an XFEL beam can therefore be tuned by a factor of about one million. The SASE 1 undulator will deliver $10^{12}$ photons in an ultra-short pulse of $100 \mathrm{fs}$ duration (the timescale of molecular vibrations), yielding a peak power of about $20 \mathrm{GW}$ at a photon energy $\mathrm{E} \approx 12 \mathrm{KeV}$ (the photon wavelength is $\lambda=h c / \mathrm{E} \approx 0.1 \mathrm{~nm}$ ).

Recall that all photons in a single micropulse are completely coherent. Since each pulse contains $\sim 10^{12}$ photons and a few hundred micropulses, there are $10^{9}$ indistinguishable ("degenerate") photons in the coherence volume. In comparison, the degeneracy parameter $D \approx 0.03$ at a synchrotron source with $\lambda=0.1 \mathrm{~nm}$. Because of the large transverse coherence area of $70 \times 70 \mu \mathrm{m}^{2}$ and the large number of coherent photons per pulse, an interference ("speckle") pattern can be recorded with a single XFEL pulse [44]. Therefore, X-ray FELs can be used not only to probe the structure of matter down to the size of an atom, but also to take 'snapshots' of the motion of atoms and molecules [45].

\section{Summary}

The main "bottleneck" limiting the beam power in circular machines is caused by space charge effects that produce beam instabilities. Such a "bottleneck" exists at the J-PARC proton synchrotron complex, and is also intrinsic to the "proton drivers" envisaged at CERN and Fermilab. In order to maximally increase the beam power of a "proton driver", it is proposed to build a facility consisting of a low-energy injector linac (PI) and a high-energy pulsed superconducting L-band linac (SCL). The $2.5 \mathrm{GeV}$ PI, based on the European Spallation Source (ESS) linac, would serve both as an injector to the SCL and a source of proton beams that could be used to copiously produce muons and "cold" neutrons. Protons

${ }^{4}$ The occurrence of higher harmonics is explained in [38]. In the forward region $(\theta=0)$ of a planar undulator, only the odd higher harmonics are observed, while the off-axis radiation contains also the even harmonics. For the European XFEL, simulations predict that the relative contribution to the total radiation power of the $3^{\text {rd }}$ and the $5^{\text {th }}$ harmonic is about $1 \%$ and $0.03 \%$, respectively [ 41 ]. 
accelerated by the SCL to $20 \mathrm{GeV}$ would be transferred through the KEK Tristan ring in order to create neutrino, kaon and muon beams for fixed-target experiments. At a later stage, a $70 \mathrm{GeV}$ proton synchrotron could be installed inside the Tristan ring. The proposed facility would be constructed using the existing KEK accelerator infrastructure and the most advanced linac technologies currently available.

Experiments with high-intensity neutrino beams are designed primarily to explore the mass spectrum of the neutrinos and their properties under the $\mathrm{CP}$ symmetry. To search for CP violation in neutrino oscillations, a $100 \mathrm{kt}$ water Cherenkov detector could be built at Okinoshima, located at a distance $\mathrm{L}_{2} \approx 650 \mathrm{~km}$ from KEK. Using the proposed KEK “proton driver", the detector at Okinoshima and the existing 0.022 Mt Super-Kamiokande detector (placed $\mathrm{L}_{1} \approx 300 \mathrm{~km}$ away from $\mathrm{KEK}$ ), the neutrino mass hierarchy could be determined either by comparing the $v_{e}$ appearance probabilities measured at the two vastly different baseline lengths $L_{1}$ and $L_{2}$, or by measuring at $L_{1}$ and $\mathrm{L}_{2}$ the neutrino energy of the first oscillation maximum. Once the mass hierarchy is determined, the $\mathrm{CP}$-violating phase in the mixing matrix can be measured with a precision of $\pm 20^{\circ}$, assuming that $2.5 \times 10^{21}$ protons are delivered on target for both $v_{e}$ and $\overline{v_{e}}$ beams.

Some of the most important discoveries in particle physics emerged from high-precision studies of $\mathrm{K}$ mesons ("kaons"), in particular neutral kaons. A deeper insight into CP violation is expected to be gained from measurements of ultra-rare kaon decays such as $K_{\mathrm{L}}^{0} \rightarrow \pi^{0} v \bar{\nu}$ and $K_{\mathrm{L}}^{+} \rightarrow \pi^{+} \nu \bar{\nu}$. These decays provide important information on higher-order effects in electroweak interactions, and therefore can serve as a probe of new phenomena not predicted by the Standard Model.

A unique feature of the proposed facility is the use of superconducting ILCtype cavities to accelerate both protons and electrons, which considerably increases its physics potential. Polarized electrons and positrons can be employed to study the structure of composite particles and the dynamics of strong interactions, as well as to search for new physics beyond the Standard Model.

An SCL-based X-ray free-electron laser (XFEL) and a synchrotron light source for applications in materials science and medicine are also envisaged. The ultrashort pulse duration of an XFEL matches the timescale of non-equilibrium microscopic processes, allowing the dynamics of atoms and molecules to be recorded in the form of femtosecond "movies".

\section{Acknowledgements}

For useful comments and suggestions concerning various aspects of this work, I am grateful to K. Fujii, K. Hagiwara, T. Higo, E. Kako, Y. Kamiya, K. Oide, K. Takayama and K. Tokoshuku. I wish to express my special gratitude to Kaoru Yokoya for his invaluable help and encouragement.

This paper is an abridged version of KEK Preprint 2014-35 (2014), which was subsequently published as an arXiv e-print (arXiv:1411.4874). 


\section{References}

[1] Aad, G., et al. (2012) Observation of a New Particle in the Search for the Standard Model Higgs Boson with the ATLAS Detector at the LHC. Physics Letters B, 716, 1-29. https://doi.org/10.1016/j.physletb.2012.08.020

[2] Chatrchyan, S., et al. (CMS Collab.) (2012) Observation of a New Boson at a Mass of $125 \mathrm{GeV}$ with the CMS Experiment at the LHC. Physics Letters B, 716, 30-61. https://doi.org/10.1016/j.physletb.2012.08.021

[3] Yano, A., et al. (2004) The Toshiba E3736 Multi-Beam Klystron. Proceedings of LINAC 2004, Lubeck, Germany, 706-708.

[4] Gelvich, E., et al. (1993) The New Generation of High-Power Multiple-Beam Klystrons. IEEE Transactions on Microwave Theory and Techniques, 41, 15-19. https://doi.org/10.1109/22.210224

[5] Yamamoto, A. (2009) Superconducting RF Cavity Development for the International Linear Collider. IEEE Trans. Appl. Supercond., 19, 1387-1393.

[6] Ostroumov, P.N. (2006) Physics Design of the $8 \mathrm{GeV}$ H-Minus Linac. New Journal of Physics, 8, 281. https://doi.org/10.1088/1367-2630/8/11/281

[7] Reschke, D. (2013) Infrastructure, Methods and Test Results for the Testing of 800 Series Cavities for the European XFEL. Proceedings of SRF 2013, Paris, France, 812815.

[8] Faircloth, D. (2013) Ion Sources for High-Power Hadron Accelerators. arXiv: 1302.3745

[9] Kapchinsky, I.M. and Tepliakov, V.A. (1970) Linear Ion Accelerator with Spatially Homogenous Focusing. Pribory i Tekhnika Eksperimenta, 2, 19-22.

[10] Vretenar, M. (2012) Low-Beta Structures. CERN Yellow Report 2011-007, 319-340. arXiv:1201.2593

[11] Danared, H., Lindroos, M. and Theroine, C. (2014) ESS: Neutron Beams at the High-Intensity Frontier. CERN Courier, June 2014, 21-24.

[12] Garoby, R., et al. (2013) Proton Drivers for Neutrino Beams and Other High Intensity Applications. Journal Physics. Conference Series, 408, 012016. https://doi.org/10.1088/1742-6596/408/1/012016

[13] Baussan, E., et al. (2014) A very Intense Neutrino Super Beam Experiment for Leptonic CP Violation Discovery Based on the European Spallation Source Linac. Nuclear Physics B, 885, 127-149. https://doi.org/10.1016/j.nuclphysb.2014.05.016

[14] Al-Binni, U., et al. (2013) Project-X, Physics Opportunities. arXiv:1306.5009v2

[15] Dudek, J., et al. (2012) Physics Opportunities with the $12 \mathrm{GeV}$ Upgrade at Jefferson Lab. arXiv:1208.1244v2

[16] Voutier, E. (2014) Physics Perspectives at JLab with a Polarized Positron Beam. arXiv:1402.2814v1

[17] Belusevic, R. (2008) Relativity, Astrophysics and Cosmology. Wiley-VCH Verlag., Weinheim.

[18] Shaposhnikov, M. (2009) Baryon Asymmetry of the Universe and Neutrinos. Progress of Theoretical Physics, 122, 185-203. https://doi.org/10.1143/PTP.122.185

[19] Hagiwara, K., et al. (2012) Physics Potential of Neutrino Oscillation Experiment with a Far Detector in Oki Island along the T2K Baseline, KEK-TH-1568. arXiv:1209.2763v1

[20] Back, J., et al. (2013) Particle Production and Energy Deposition Studies for the Neutrino Factory Target Station. Physical Review Accelerators and Beams, 16, 021001. https://doi.org/10.1103/PhysRevSTAB.16.021001 
[21] McDonald, K.T., et al. (2010) The MERIT High-Power Target Experiment at the CERN PS. Proceedings of First International Particle Accelerator Conference, Kyoto, Japan, 3527-3529.

[22] Baussan, E., et al. (2013) Target, Magnetic Horn and Safety Studies for the CERN to Frejus Super Beam. Journal of Physics: Conference Series, 408, 012061. https://doi.org/10.1088/1742-6596/408/1/012061

[23] Kamiya, Y. DC Neutrino Horn. The manuscript (in Japanese) has not been published.

[24] Omori, T., et al. (2006) Efficient Propagation of Polarization from Laser Photons to Positrons through Compton Scattering and Electron-Positron Pair Creation. Physical Review Letters, 96, 114801. https://doi.org/10.1103/PhysRevLett.96.114801

[25] Grames, J., et al. (2013) PEPPo: Using a Polarized Electron Beam to Produce Polarized Positrons. $15^{\text {th }}$ International Workshop on Polarized Sources, Targets and Polarimetry, Charlottesville, Virginia, USA.

[26] Zel'dovich, Y.B. (1959) Parity Nonconservation in the First Order in the Weak-Interaction Constant in Electron Scattering and Other Effects. Journal of Experimental and Theoretical Physics (JETP), 36, 964-966.

[27] Derman, E. and Marciano, W.J. (1979) Parity Violating Asymmetries in Polarized Electron Scattering. Annals of Physics, 121, 147-180.

[28] Czarnecki, A. and Marciano, W.J. (1996) Electroweak Radiative Corrections to Polarized Moller Scattering Asymmetries. Physical Review D, 53, 1066-1072. https://doi.org/10.1103/PhysRevD.53.1066

[29] Mammei, J. (2012) The MOLLER Experiment. Nuovo Cimento, 35, 203-208.

[30] Kumar, K.S., et al. (2013) Low-Energy Measurements of the Weak Mixing Angle. Annual Review of Nuclear and Particle Science, 63, 237-267. https://doi.org/10.1146/annurev-nucl-102212-170556

[31] Belusevic, R. (1999) Neutral Kaons. Springer-Verlag, Berlin.

[32] Brod, J., Gorbahn, M. and Stamou, E. (2011) Two-Loop Electroweak Corrections for the $K \rightarrow \pi v \bar{v}$ Decays. Physical Review D, 83, 034030. https://doi.org/10.1103/PhysRevD.83.034030

[33] Belyaev, A., et al. (2001) Kaon Physics with a High Intensity Proton Driver, CERNTH/2001-175. arXiv:hep-ph/0107046v2

[34] Buras, A. (1999) CP Violation and Rare Decays of K and B Mesons. arXiv:hep-ph/9905437

[35] Artamonov, A., et al. (2009) Study of the Decay $K^{+} \rightarrow \pi^{+} v \bar{v}$ in the Momentum Region $140<\mathrm{P}_{\pi}<199 \mathrm{MeV} /$ c. Physical Review D, 79, 092004. https://doi.org/10.1103/PhysRevD.79.092004

[36] Miller, J., de Rafael, E. and Roberts, B.L. (2007) Muon (g-2): Experiment and Theory. Reports on Progress in Physics, 70, 795-881. https://doi.org/10.1088/0034-4885/70/5/R03

[37] Mibe, T. (2010) New g-2 Experiment at J-PARC. Chinese Physics C, 34, 745-748.

[38] Schmuser, P., Dohlus, M. and Rossbach, J. (2008) Ultraviolet and Soft X-Ray FreeElectron Lasers. Springer-Verlag, Berlin.

[39] Huang, Zh. and Kim, K. (2007) Review of X-Ray Free-Electron Laser Theory. Physical Review Accelerators and Beams, 10, 034801. https://doi.org/10.1103/PhysRevSTAB.10.034801

[40] Amann, J., et al. (2012) Demonstration of Self-Seeding in a Hard-X-Ray Free-Electron Laser. Nature Photonics, 6, 693-698. https://doi.org/10.1038/nphoton.2012.180 
[41] Abela, R., et al. (2007) The European X-Ray Free-Electron Laser. Technical Design Report, DESY 2006-097.

[42] Arthur, J. (1999) Prospects for an X-Ray FEL Light Source and Some Possible Scientific Applications, SLAC-PUB-8276.

[43] Agapov, I., et al. (2014) SASE Characteristics from Baseline European XFEL Undulators in the Tapering Regime. Proceedings of FEL 2014, Basel, Switzerland, 159163.

[44] Pellegrini, C. and Stohr, J. (2003) X-Ray Free-Electron Lasers-Principles, Properties and Applications. Nuclear Instruments and Methods in Physics Research Section A: Accelerators, Spectrometers, Detectors and Associated Equipment, 500, 33-40. https://doi.org/10.1016/S0168-9002(03)00739-3

[45] Barty, A. (2010) Time-Resolved Imaging Using X-Ray Free Electron Lasers. Journal of Physics B: Atomic, Molecular and Optical Physics, 43, 194014. https://doi.org/10.1088/0953-4075/43/19/194014

\section{Scientific Research Publishing}

Submit or recommend next manuscript to SCIRP and we will provide best service for you:

Accepting pre-submission inquiries through Email, Facebook, LinkedIn, Twitter, etc. A wide selection of journals (inclusive of 9 subjects, more than 200 journals)

Providing 24-hour high-quality service

User-friendly online submission system

Fair and swift peer-review system

Efficient typesetting and proofreading procedure

Display of the result of downloads and visits, as well as the number of cited articles

Maximum dissemination of your research work

Submit your manuscript at: http://papersubmission.scirp.org/

Or contact jamp@scirp.org 